\title{
Mathematical solution of multilevel fractional programming problem with fuzzy goal programming approach
}

\author{
Kailash Lachhwani ${ }^{*}$ and Mahaveer Prasad Poonia ${ }^{2}$
}

\begin{abstract}
In this paper, we show a procedure for solving multilevel fractional programming problems in a large hierarchical decentralized organization using fuzzy goal programming approach. In the proposed method, the tolerance membership functions for the fuzzily described numerator and denominator part of the objective functions of all levels as well as the control vectors of the higher level decision makers are respectively defined by determining individual optimal solutions of each of the level decision makers. A possible relaxation of the higher level decision is considered for avoiding decision deadlock due to the conflicting nature of objective functions. Then, fuzzy goal programming approach is used for achieving the highest degree of each of the membership goal by minimizing negative deviational variables. We also provide sensitivity analysis with variation of tolerance values on decision vectors to show how the solution is sensitive to the change of tolerance values with the help of a numerical example.
\end{abstract}

Keywords: Multilevel fractional programming, Fuzzy goal programming, Membership function, Tolerance values

\section{Background}

Hierarchical optimization or multilevel programming problems (MLPPs) have the following common characteristics: interactive decision making units exist within predominantly hierarchical structures; the execution of decision is sequential from higher level to lower level; each decisionmaking unit independently controls a set of decision variables and is interested in maximizing its own objective but is affected by the reaction of lower level decision makers (DMs). Due to their dissatisfaction with the decision of the higher level DMs, decision deadlock arises frequently in the decision-making situation. Multilevel fractional programming problems (MLFPPs) involve objective functions in fractional form, i.e., $f(X)=\frac{N(X)}{D(X)}$ at each level with the assumption that the denominator of objectives remains positive at each level in the feasible region. Some important existing solution approaches such as the extreme point search, the procedure based on the Karush-Kuhn Tucker condition, and the decent method (Anandilingam 1988;

\footnotetext{
* Correspondence: kailashclachhwani@yahoo.com

${ }^{1}$ Department of Mathematics, Government Engineering College, Bikaner 334004, India

Full list of author information is available at the end of the article
}

Anandilingam and Apprey 1991; Biswas and Pal 2005; Bellmann 1957; Charnes and Cooper 1962; Craven and Mond 1975; Lai 1996) are effective only for solving simple types of multilevel programming problems. Initially, fuzzy approach was used to handle multiobjective optimization problems (Chakraborty and Gupta 2002; Jimenez and Bilbas 2009). Lai and Hwang (1993) at first developed an effective fuzzy approach using the concept of tolerance membership functions for solving MLPPs in 1996. Shih et al. (1996) extended Lai's concept using a non-compensatory maximum-minimum aggregation operator for solving MLPPs. Shih and Lee (2000) further extended Lai's concept by introducing the compensatory fuzzy operator for solving MLPPs. Sinha (2003a,b) studied alternative MLP techniques based on fuzzy mathematical programming (FMP). The basic concept of these fuzzy approaches is the same, and evaluation of the problem again and again by redefining the elicited membership values is essentially needed in the solution search process to obtain a satisfactory solution. So, computational load is also inherently involved in the fuzzy approaches developed so far. In the FMP techniques of Sinha (2003a,b), the last (lower) level is the most important, and the decision of the lowest level remains either unchanged or closest to individual best decisions, which leads 
to the paradox that the decision power of the lowest level DM dominates the higher level DM. To overcome such difficulties, the fuzzy goal programming (FGP) approach to multidecision-making problems was introduced by Mohamed (1997) which is extended by Pramanik and Roy (2007) to solve MLPPs. Baky (2009) used fuzzy goal programming to solve decentralized bilevel multiobjective programming problems. Chang (2009) suggested goal programming approach for fuzzy multiobjective fractional programming problems. Recently, Pal and Gupta (2009) studied a genetic algorithm to fuzzy goal programming formulation of fractional multiobjective decision-making problems.

In real-world decision-making situations, decision makers sometimes may be faced with the decision to optimize inventory/sales, actual cost/standard cost, output/employee, etc. with respect to some constraints. Such type of problems in a large hierarchical organization from higher level to lower level and their sequential decisions on complex and conflicting objectives formulate the MLFPPs. Practical optimization situations involving multilevel with fractional objectives have been rare, but such problems can be encountered in the most complex design, pattern recognition, control theory, and resource allocation situations (Mohamed 1997). Motivated by the concept of interactive fuzzy goal programming and fractional programming, an effort has been made to examine the possibility of unifying the level-wise (hierarchical) and stage-wise operations with the assumption of a positive denominator of objective functions at each level. The aim of this paper is to present a procedure to solve multilevel fractional programming problems. Our proposed methodology involves the fuzzy goal levels of the numerator and denominator part of each objective as well as decision vectors controlled by the higher level DMs, which are determined by individual optimal solutions. Then, the fuzzy goals are characterized by the associated membership functions which are transformed into fuzzy flexible membership goals by means of introducing negative and positive deviational variables and assigning a higher membership value (unity) as aspiration level to each of them. Since overdeviation from any fuzzy goal implies the full achievement of the membership values, we assign only negative deviational variables to the achievement function and minimize negative deviational variables to get a compromise optimal solution. To illustrate our proposed method, we solve a numerical example and compare the results with the change in tolerance limits.

The paper is organized as follows: In the 'Formulation of MLPP' section, we discuss formulation of MLFPP and the related terminology. In the 'Fuzzy programming formulation of MLFPP' section, we characterize the linear membership functions for the numerator and denominator of objective functions at each level as well as decision vectors controlled by the higher level DMs. In the next section, we discuss the proposed FGP approach to tackle MLFPPs and formulate different mathematical models related to it. In the 'Selection of compromise solution' section, selection criteria of compromise optimal solution are described. To illustrate the proposed methodology, a numerical example is considered and sensitivity analysis is performed with the change in tolerance limits in the 'Numerical example' section. Concluding remarks are given in the last sections.

\section{Results and discussion \\ Formulation of MLFPP}

We consider a T-level fractional programming problem of maximization-type objectives at each level. Mathematically, we can state it as follows:

$$
\begin{aligned}
& \operatorname{Max}_{\bar{X}_{1}} Z_{1}(\bar{X})=\frac{\overline{C_{11}} \bar{X}_{1}+\overline{C_{12}} \overline{X_{2}}+\ldots+\overline{C_{1 T}} \bar{X}_{T}+\alpha_{1}}{\overline{D_{11}} \bar{X}_{1}+\bar{D}_{12} \bar{X}_{2}+\ldots+\bar{D}_{1 T} \bar{X}_{T}+\beta_{1}} \\
& \operatorname{Max}_{\overline{\mathrm{X}}_{2}} Z_{2}(\bar{X})=\frac{\overline{C_{21}} \bar{X}_{1}+{\overline{C_{22}}}_{\overline{X_{2}}}+\ldots+\bar{C}_{2 T} \bar{X}_{T}+\alpha_{2}}{\overline{\bar{D}_{21}} \bar{X}_{1}+\bar{D}_{22} \bar{X}_{2}+\ldots .+\bar{D}_{2 T} \bar{X}_{T}+\beta_{2}} \\
& \underset{\overline{\mathrm{X}}_{\mathrm{T}}}{\operatorname{Max}} Z_{T}(\bar{X})=\frac{\bar{C}_{T 1} \overline{X_{1}}+\overline{C_{T 2}} \overline{X_{2}}+\ldots+\overline{C_{T T}} \bar{X}_{T}+\alpha_{T}}{\bar{D}_{T 1} \overline{X_{1}}+\overline{D_{T 2}} \bar{X}_{2}+\ldots+\overline{D_{T T}} \overline{X_{T}}+\beta_{T}}
\end{aligned}
$$

subject to

$$
\overline{\mathrm{A}}_{\mathrm{i} 1} \bar{X}_{1}+\overline{\mathrm{A}}_{\mathrm{i} 2} \bar{X}_{2}+\ldots \ldots \ldots+\begin{aligned}
& \overline{\mathrm{A}}_{\mathrm{iT}} \bar{X}_{T}(\leq,=, \geq) b_{i} \\
& \forall i=1,2, \ldots ., m
\end{aligned}
$$$$
\text { and }
$$

$\bar{X}_{1} \geq 0, \bar{X}_{2} \geq 0, \ldots, \bar{X}_{T} \geq 0$

$\bar{X}_{1}=\left\{X_{1}^{1}, X_{1}^{2}, \ldots \ldots . ., X_{1}^{N_{1}}\right\}^{\prime}$ decision variables are under the control of the first level DM; $\bar{X}_{T}=\left\{X_{T}^{1}, X_{T}^{2}, \ldots \ldots . ., X_{T}^{N_{T}}\right\}^{\prime}$ decision variables are under the control of the t-level DM.

Where ' denotes transposition, $\bar{A}_{i j} i=1,2, \ldots ., m$, and $j=1,2, \ldots, T$ are $m$ row vectors, each with dimension $\left(1 \times N_{j}\right) \cdot \overline{\mathrm{A}}_{\mathrm{it}} \bar{X}_{t}, t=1,2, \ldots, T$ is a column vector of dimension $(M \times 1) . \bar{C}_{11}, \bar{C}_{21}, \ldots, \bar{C}_{T 1}$ are row vectors of dimension $\left(1 \times N_{1}\right)$. Similarly, $\overline{C_{1 T}}, \overline{C_{2 T}}, \ldots, \overline{C_{T T}}$ and $\overline{D_{1 T}}, \overline{D_{2 T}}, \ldots, \overline{D_{T T}}$ are row vectors of dimension $\left(1 \times N_{T}\right)$. We take $\bar{X}=\bar{X}_{1} \cup \bar{X}_{2} \cup \ldots \cup \bar{X}_{T}$ and $N=N_{1}+N_{2}+\ldots \ldots .+$ $N_{T}$. Here, one DM is located on each level. Decision vector $\bar{X}_{t}, t=1,2, \ldots, T$ is the control of the $\mathrm{t}$-th level DM having $N_{t}$ number of decision variables. Here, it is assumed that the denominator of objective functions is positive at each level for all the values of decision variables in the constraint region.

\section{Fuzzy programming formulation of MLFPP}

To formulate the fuzzy programming model of MLFPP, the objective numerator $f_{i N}(\bar{X})+\alpha_{i}, \forall t=1,2, \ldots, T$ and objective denominator $f_{i D}(\bar{X})+\alpha_{i}, \quad \forall t=1,2, \ldots, T$ at 
each level and the decision vector $\bar{X}_{t},(t=1,2, . ., T-1)$ would be transformed into fuzzy goals by means of assigning an aspiration level to each of them. Then, they are to be characterized by the associated membership functions by defining tolerance limits for the achievement of the aspired levels of the corresponding fuzzy goals.

\section{Characterization of membership function of MLFPP}

In the decision-making context, each DM is interested in maximizing his or her own objective function; the optimal solution of each DM when calculated in isolation would be considered as the best solution, and the associated objective values can be considered as the aspiration level of the corresponding fuzzy goal. Let $\bar{X}_{t}^{B}$ be the best solution of the $\mathrm{t}$-th level DM. It is quite natural that objective values which are equal to or larger than $Z_{t}^{B}=Z_{t}\left(\bar{X}_{t}^{B}\right)=\underset{\bar{X} \in X}{\operatorname{Max}} Z_{t}(\bar{X}), t=1$, $2, \ldots, T$ should be absolutely satisfactory to the $\mathrm{t}$-th level DM. If the individual best solutions $\bar{X}_{t}^{B}, t=1,2, \ldots, T$ are the same, then a satisfactory optimal solution of the system is reached. However, this rarely happens due to the conflicting nature of the objectives. To obtain a satisfactory solution, the higher DM should give some tolerance (relaxation), and the relaxation of the decision of the higher level DM depends on the needs, desires, and practical situations in the decision-making situation. Then, the fuzzy goals take the form

$Z_{t}(\bar{X}) \geq Z_{t}\left(\bar{X}_{t}^{B}\right), t=1,2, \ldots, T$

and $\bar{X}_{t} \cong \bar{X}_{t}^{B}, t=1,2, \ldots,(T-1)$.

To build membership functions, fuzzy goals and tolerance should be determined first. However, they could hardly be determined without meaningful supporting data. Using the individual best solution, we find the values of all the numerator objective functions and denominator objective functions at each best solution and construct a payoff matrix as follows:

$$
\left[\begin{array}{cccccc}
X_{1}^{B} & N_{1} & N_{2} & \cdot & \cdot & N_{T} \\
\overline{X_{1}^{B}} & N_{1}\left(\bar{X}_{1}^{B}\right) & N_{2}\left(\bar{X}_{1}^{B}\right) & \cdot & \cdot & N_{T}\left(\bar{X}_{1}^{B}\right) \\
\overline{X_{2}^{B}} & N_{1}\left(\bar{X}_{2}^{B}\right) & N_{2}\left(\bar{X}_{2}^{B}\right) & \cdot & \cdot & N_{T}\left(\bar{X}_{2}^{B}\right) \\
\dot{\cdot} & \cdot \bar{\cdot} & \cdot & \cdot & \\
\overline{X_{T}^{B}} & N_{1}\left(\bar{X}_{T}^{B}\right) & N_{2}\left(\bar{X}_{T}^{B}\right) & \cdot & \cdot & N_{T}\left(\bar{X}_{T}^{B}\right)
\end{array}\right]
$$

and

$$
\left[\begin{array}{cccccc}
X_{1}^{B} & D_{1} & D_{2} & \cdot & \cdot & D_{T} \\
\overline{X_{1}^{B}} & D_{1}\left(\bar{X}_{1}^{B}\right) & D_{2}\left(\bar{X}_{1}^{B}\right) & \cdot & \cdot & D_{T}\left(\bar{X}_{1}^{B}\right) \\
\overline{X_{2}^{B}} & D_{1}\left(\bar{X}_{2}^{B}\right) & D_{2}\left(\bar{X}_{2}^{B}\right) & \cdot & \cdot & D_{T}\left(\bar{X}_{2}^{B}\right) \\
\cdot & \cdot & \cdot & \cdot & \cdot & \cdot \\
\bar{X}_{T}^{B} & D_{1}\left(\bar{X}_{T}^{B}\right) & D_{2}\left(\bar{X}_{T}^{B}\right) & \cdot & \cdot & \\
\cdot\left(\overline{X_{T}^{B}}\right)
\end{array}\right]
$$

Here, $\bar{X}_{t}(t=1,2, \ldots, T)$ are assumed to be the main decision vectors. The maximum value of each column $N_{t}\left(\bar{X}_{t}^{B}\right)$ and $D_{t}\left(\bar{X}_{t}^{B}\right)$ give upper tolerance limit or aspired level of achievement for the $t$-th numerator objective function and denominator objective function, respectively, where $N_{t}^{B}=N_{t}\left(\bar{X}_{t}^{B}\right)=\operatorname{Max}_{\bar{X} \in X} N_{t}(\bar{X}), t=1,2, \ldots, T$. The minimum value of each column gives the lower tolerance limit or lowest acceptable level of achievement for the $\mathrm{t}$-th numerator objective function and denominator objective function, respectively, where $N_{t}^{L}=\operatorname{Min}_{\bar{X} \in X}\left\{N_{t}\left(\bar{X}_{1}^{B}\right)\right.$, $\left.N_{t}\left(\bar{X}_{2}^{B}\right), \ldots, N_{t}\left(\bar{X}_{T}^{B}\right)\right\}, . t=1,2, \ldots, T$. Then, the linear membership functions for the defined fuzzy goals are as follows (see also Figures 1 and 2):

$$
\mu_{Z_{t}}\left(N_{t}(\bar{X})\right)=\left\{\begin{array}{cl}
1 & \text { if } \mathrm{N}_{t}(\bar{X}) \geq N_{t}^{B} \\
\frac{N_{t}(\bar{X})-N_{t}^{L}}{N_{t}^{B}-N_{t}^{L}} & \text { if } \mathrm{N}_{t}^{L} \leq N_{t}(\bar{X}) \leq N_{t}^{B} \\
0 & \text { if } \mathrm{N}_{t}(\bar{X}) \leq N_{t}^{L} \\
& \forall t=1,2, \ldots, T
\end{array}\right.
$$

(a)

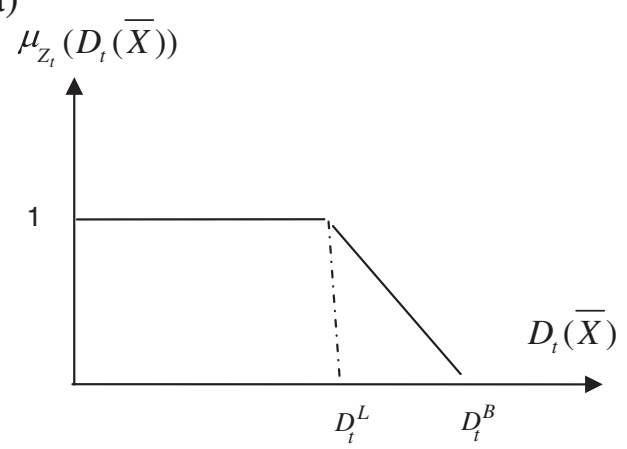

(b)

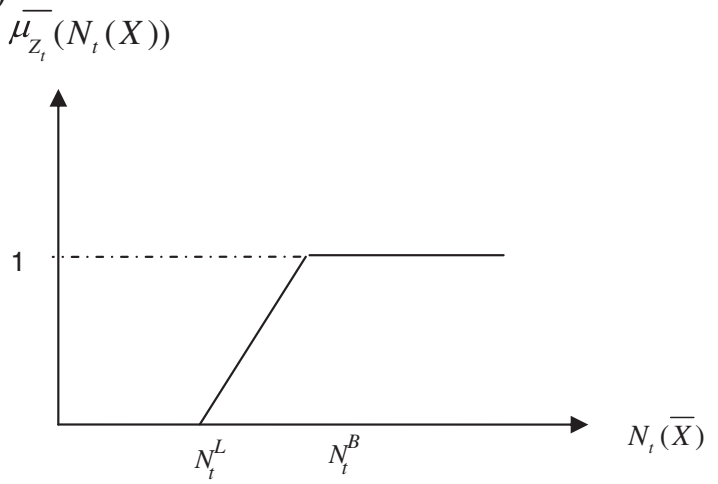

Figure 1 (a): Membership function for $\mu_{\mathrm{z}_{t}}\left(D_{i}(\bar{X})\right)$ (b): Membership function for $\mu_{Z_{t}}\left(N_{i}(\bar{X})\right)$. 


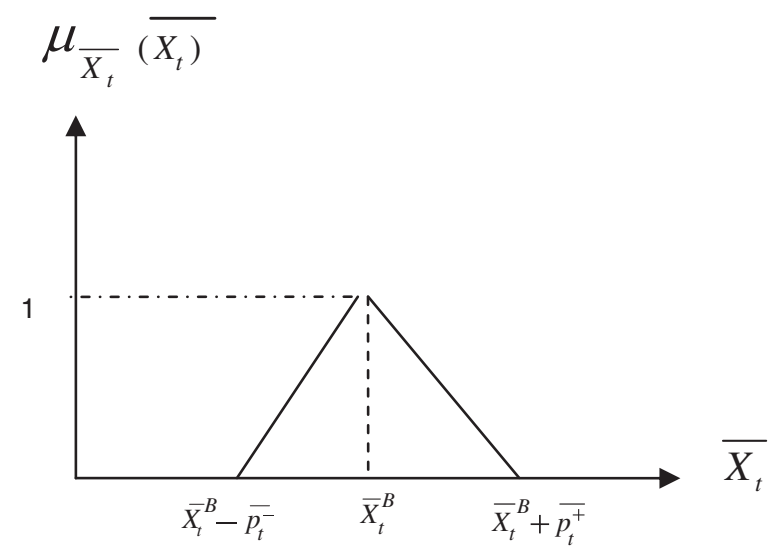

Figure 2 Membership functions of decision vector $\bar{X}_{t}(t=1,2, \ldots$, $\mathrm{T}-1)$.

$\mu_{Z_{t}}\left(D_{t}(\bar{X})\right)=\left\{\begin{array}{cl}0 & \text { if } \mathrm{D}_{t}(\bar{X}) \geq D_{t}^{B} \\ \frac{D_{t}^{B}-D_{t}(\bar{X})}{D_{t}^{B}-D_{t}^{L}} & \text { if } \mathrm{D}_{t}^{L} \leq D_{t}(\bar{X}) \leq D_{t}^{B} \\ 1 & \text { if } \mathrm{D}_{t}(\bar{X}) \leq D_{t}^{L} \\ & \forall t=1,2, \ldots, T\end{array}\right.$

Here, linear membership functions are more suitable than nonlinear functions as less computational difficulties arise in models due to it. Let $\overline{p_{t}^{-}}, \overline{p_{t}^{+}}(t=1,2, \ldots, T-1)$ be the negative and positive tolerance values on decision vectors $\bar{X}_{t}$ considered by the t-th level DM. This is a triangular fuzzy number. Then, the linear membership functions for decision vectors $\bar{X}_{t}$ can be formulated as follows:

$\mu_{\bar{X}_{t}}\left(\bar{X}_{t}\right)=\left\{\begin{array}{cc}\frac{\overline{X_{t}}-\left(\bar{X}_{t}^{B}-\overline{p_{t}^{-}}\right)}{\overline{p_{t}^{-}}} \text {if }\left(\bar{X}_{t}^{B}-\overline{p_{t}^{-}}\right) \leq \bar{X}_{t} \leq \bar{X}_{t}^{B} \\ \frac{\left(\bar{X}_{t}^{B}+\overline{p_{t}^{+}}\right)-\bar{X}_{t}}{\overline{p_{t}^{+}}} \text {if } \bar{X}_{t}^{B} \leq \bar{X}_{t} \leq\left(\bar{X}_{t}^{B}+\overline{p_{t}^{+}}\right) \\ 0 \quad \text { otherwise } \\ \forall t=1,2, \ldots, T\end{array}\right.$

Here, $\overline{p_{t}^{-}}$and $\overline{p_{t}^{+}}$are the negative and positive tolerance vectors; $\overline{p_{t}^{-}}$and $\overline{p_{t}^{+}}$are not necessarily same. Generally, $\bar{X}_{t}$ lies between $\bar{X}_{t}^{B}-\overline{p_{t}^{-}}$and $\bar{X}_{t}^{B}+\bar{p}_{t}^{+}$. DMs may prefer to shift the range of $\bar{X}_{t}^{B}$ which may be the left of $\bar{X}_{t}^{B}$ or the right of $\bar{X}_{t}^{B}$, only depending on the needs and desires of the higher level DMs in the decision-making situation. Then, the membership function becomes one-sided. For example, if $\bar{X}=\overline{0}$, then $\bar{X}$ should lie on the right of $\overline{0}$. Then, the DM should assign $\overline{p_{t}^{-}} \leq \overline{0}, \overline{p_{t}^{+}} \geq \overline{0}$ and $\left|\overline{p_{t}^{-}}\right| \leq\left|\overline{p_{t}^{+}}\right|$. If the DM wants the shift towards the left of $\bar{X}_{t}^{B}$, then $\overline{p_{t}^{-}}$should be assigned a positive value while $\overline{p_{t}^{+}}$ should be assigned a negative value, i.e., $\overline{p_{t}^{-}} \geq \overline{0}, \overline{p_{t}^{+}} \leq \overline{0}$, and $\left|\overline{p_{t}^{-}}\right| \geq\left|\overline{p_{t}^{+}}\right|$. Similarly, if the shift is required to the right of $\bar{X}_{t}^{B}$, then the DM should assign $\overline{p_{t}^{-}} \leq \overline{0}, \overline{p_{t}^{+}} \geq 0$, and $\left|\overline{p_{t}^{-}}\right| \leq\left|\overline{p_{t}^{+}}\right|$. We may treat the tolerance as variables with the restrictions that $\overline{p_{t}^{-}} \leq \bar{X}_{t}^{B}$ (so that the value of the variables remain non-negative).

\section{FGP solution approach}

FGP is an extension of conventional goal programming (GP) introduced by Charnes and Cooper (1962). GP has been extensively studied and widely circulated in literature (Arora and Gupta 2009; Pramanik and Roy 2007). In this paper, GP approach to fuzzy multiobjective decision-making problems introduced by Mohamed (1997) is extended to solve MLFPP problems. In a decision-making situation, the aim of each DM is to achieve the highest membership value (unity) of the associated fuzzy goal in order to obtain the absolute satisfactory solution. However, in real practice, achievement of all membership values to the highest degree (unity) is not possible due to conflicting objectives. Therefore, the decision policy for minimizing the regrets of the DMs for all the levels should be taken into consideration. Then, each DM should try to maximize his or her membership function by making them as close as possible to unity by minimizing its negative deviational variables. Therefore, in effect, we are simultaneously optimizing all the objective functions. So, for the defined membership functions in Equations 3, 4, and 5, the flexible membership goals having the aspired level unity can be represented as follows:

$\mu_{Z_{t}}\left(N_{t}(\bar{X})\right)+D_{t 1}^{-}-D_{t 1}^{+}=1 ; t=1,2, \ldots, T$

$\mu_{Z_{t}}\left(D_{t}(\bar{X})\right)+D_{t 2}^{-}-D_{t 2}^{+}=1 ; t=1,2, \ldots, T$

$\mu_{\bar{X}_{t}}\left(\bar{X}_{t}\right)+\bar{D}_{t 3}^{-}-\bar{D}_{t 3}^{+}=\bar{I} ; t=1,2, \ldots,(T-1)$

Here, $D_{t 1}^{-}, D_{t 2}^{-}$are negative deviational variables, and $D_{t 1}^{+}, D_{t 2}^{+}$are positive deviational variables; $\bar{D}_{t 3}^{+}, \bar{D}_{t 3}^{-}$ represent the vector of negative deviational and positive deviational variables. It is to be noted that any overdeviation from a fuzzy goal implies the full achievement value. Then, Equations 6, 7, and 8 can be written as follows:

$\mu_{Z_{t}}\left(N_{t}(\bar{X})\right)+D_{t 1}^{-} \geq 1 ; t=1,2, \ldots, T$

$\mu_{Z_{t}}\left(D_{t}(\bar{X})\right)+D_{t 2}^{-} \geq 1 ; t=1,2, \ldots, T$

$\mu_{\bar{X}_{t}}\left(\bar{X}_{t}\right)+\bar{D}_{t 3}^{-} \geq \bar{I} ; t=1,2, \ldots,(T-1)$ 
FGP formulation can be presented as follows: Model I: Find $\bar{X}$ so as to minimize $\lambda$ subject to

$$
\begin{aligned}
& \mu_{Z_{t}}\left(N_{t}(\bar{X})\right)+D_{t 1}^{-} \geq 1 ; t=1,2, \ldots, T \\
& \mu_{Z_{t}}\left(D_{t}(\bar{X})\right)+D_{t 2}^{-} \geq 1 ; t=1,2, \ldots, T \\
& \mu_{\bar{X}_{t}}\left(\bar{X}_{t}\right)+\bar{D}_{t 3}^{-} \geq \bar{I} ; t=1,2, \ldots,(T-1) \\
& \lambda \geq D_{t 1}^{-} ; t=1,2, \ldots, T \\
& \lambda \geq D_{t 2}^{-} ; t=1,2, \ldots, T \\
& \lambda \geq \bar{D}_{t 3}^{-} \bar{I} ; t=1,2, \ldots,(T-1) \\
& D_{t 1}^{-} \geq 0 ; t=1,2, \ldots, T \\
& D_{t 2}^{-} \geq 0 ; t=1,2, \ldots, T \\
& \bar{D}_{t 3}^{-} \geq \overline{0}^{-} t=1,2, \ldots,(T-1) \\
& \overline{\mathrm{A}}_{\mathrm{i} 1} \bar{X}_{1}+\overline{\mathrm{A}}_{\mathrm{i} 2} \bar{X}_{2}+\ldots \ldots \ldots \ldots . .+\overline{\mathrm{A}}_{\mathrm{iT}} \bar{X}_{T}(\leq,=, \geq) b_{i} \\
& \quad \forall i=1,2, \ldots, m
\end{aligned}
$$

and $\bar{X}_{1} \geq 0, \bar{X}_{2} \geq 0, \ldots \ldots \ldots, \bar{X}_{T} \geq 0$

The above problem can be rewritten as follows: Minimize $\lambda$ subject to

$$
\begin{aligned}
& \frac{N_{t}(\bar{X})-N_{t}^{L}}{N_{t}^{B}-N_{t}^{L}}+D_{t 1}^{-} \geq 1 ; t=1,2, \ldots, T \\
& \frac{D_{t}^{B}-D_{t}(\bar{X})}{D_{t}^{B}-D_{t}^{L}}+D_{t 2}^{-} \geq 1 ; t=1,2, \ldots, T \\
& \bar{X}_{t}-\left(\bar{X}_{t}^{B}-\bar{p}_{t}^{-}\right) \bar{p}_{t}^{-}+\bar{D}_{t 31}^{-} \geq \bar{I} ; t=1,2, \ldots,(T-1) \\
& \left(\bar{X}_{t}^{B}+\bar{p}_{t}^{+}\right)-\bar{X}_{t} \bar{p}_{t}^{+}+\bar{D}_{t 32}^{-} \geq \bar{I} ; t=1,2, \ldots,(T-1) \\
& \lambda \geq D_{t 1}^{-} ; t=1,2, \ldots, T \\
& \lambda \geq D_{t 2}^{-} ; t=1,2, \ldots, T \\
& \lambda \geq \bar{D}_{t 31}^{-} \bar{I} ; t=1,2, \ldots,(T-1) \\
& \lambda \geq \bar{D}_{t 32}^{-} \bar{I} ; t=1,2, \ldots,(T-1) \\
& D_{t 1}^{-} \geq 0 ; t=1,2, \ldots, T \\
& D_{t 2}^{-} \geq 0 ; t=1,2, \ldots, T \\
& D_{t 31}^{-} \geq \overline{0} ; t=1,2, \ldots,(T-1) \\
& D_{t 32}^{-} \geq \overline{0} ; t=1,2, \ldots,(T-1) \\
& \overline{\mathrm{A}}_{\mathrm{i} 1} \bar{X}_{1}+\overline{\mathrm{A}}_{\mathrm{i} 2} \bar{X}_{2}+\ldots \ldots \ldots \ldots . .+\overline{\mathrm{A}}_{\mathrm{iT}} \bar{X}_{T}(\leq,=, \geq) b_{i} \\
& \forall i=1,2, \ldots, m
\end{aligned}
$$

and

$$
\bar{X}_{1} \geq 0, \bar{X}_{2} \geq 0, \ldots \ldots \ldots, \bar{X}_{T} \geq 0
$$

Here, $D_{t 1}^{-}, D_{t 2}^{-}$are negative deviational variables. $\bar{D}_{t 31}^{-}, \bar{D}_{t 32}^{-}$represent the vector of underdeviational variables. $\bar{I}$ is the column vector having all components equal to 1 , and its dimension depends on $\bar{X}$.

Model IIa: Find $\bar{X}$ so as to minimize

$\lambda=\sum_{t=1}^{T} W_{t 1}^{-} D_{t 1}^{-}+\sum_{t=1}^{T-1} W_{t 2}^{-} D_{t 2}^{-}+\sum_{t=1}^{T-1} \bar{W}_{t 3}^{-} \bar{D}_{t 3}^{-}$

Model IIb: Find $\bar{X}$ so as to minimize

$$
\lambda=\sum_{t=1}^{T} D_{t 1}^{-}+\sum_{t=1}^{T-1} D_{t 2}^{-}+\sum_{t=1}^{T-1} \bar{D}_{t 3}^{-}
$$

subject to

$$
\begin{aligned}
& \mu_{Z_{t}}\left(N_{t}(\bar{X})\right)+D_{t 1}^{-} \geq 1 ; \quad t=1,2, \ldots, T \\
& \mu_{Z_{t}}\left(D_{t}(\bar{X})\right)+D_{t 2}^{-} \geq 1 ; t=1,2, \ldots, T \\
& \mu_{\bar{X}_{t}}\left(\bar{X}_{t}\right)+\bar{D}_{t 3}^{-} \geq \bar{I} ; t=1,2, \ldots,(T-1) \\
& D_{t 1}^{-} \geq 0 ; \quad t=1,2, \ldots, T \\
& D_{t 2}^{-} \geq 0 ; \quad t=1,2, \ldots, T \\
& \bar{D}_{t 3}^{-} \geq \overline{0} ; \quad t=1,2, \ldots,(T-1) \\
& \overline{\mathrm{A}}_{\mathrm{i} 1} \bar{X}_{1}+\overline{\mathrm{A}}_{\mathrm{i} 2} \bar{X}_{2}+\ldots \ldots \ldots \ldots+\overline{\mathrm{A}}_{\mathrm{iT}} \bar{X}_{T}(\leq,=, \geq) b_{i} \\
& \forall i=1,2, \ldots ., m
\end{aligned}
$$

and

$\bar{X}_{1} \geq 0, \bar{X}_{2} \geq 0, \ldots \ldots \ldots, \bar{X}_{T} \geq 0$

Here, $D_{t 1}^{-}, D_{t 2}^{-}$are negative deviational variables. $\bar{D}_{t 3}^{-}$ represents the vector of underdeviational variables. The numerical weights are taken as $W_{t 1}^{-}=1 /\left(N_{t}^{B}-N_{t}^{L}\right)$, $W_{t 2}^{-}={ }^{-1} 1 /\left(D_{t}^{B}-D_{t}^{L}\right)$, and $\bar{W}_{t 3}^{-}=\left[1 /{ }_{\left(\bar{p}_{t}^{-}\right)}, 1 /{ }_{\left(\bar{p}_{t}^{+}\right)}\right] \cdot \bar{I}$ is the column vector having all components equal to 1 , and its dimension depends on $\bar{X}$. The above problem in models IIa and IIb can be rewritten as follows:

Minimize

$$
\begin{aligned}
\lambda & =\sum_{t=1}^{T} W_{t 1}^{-} D_{t 1}^{-}+\sum_{t=1}^{T-1} W_{t 2}^{-} D_{t 2}^{-}+\sum_{t=1}^{T-1} \bar{W}_{t 31}^{-} \bar{D}_{t 31}^{-} \\
& +\sum_{t=1}^{T-1} \bar{W}_{t 32}^{-} \bar{D}_{t 32}^{-}
\end{aligned}
$$

Minimize

$\lambda=\sum_{t=1}^{T} D_{t 1}^{-}+\sum_{t=1}^{T-1} D_{t 2}^{-}+\sum_{t=1}^{T-1} \bar{D}_{t 31}^{-}+\sum_{t=1}^{T-1} \bar{D}_{t 32}^{-}$

subject to

$\frac{N_{t}(\bar{X})-N_{t}^{L}}{N_{t}^{B}-N_{t}^{L}}+D_{t 1}^{-} \geq 1 ; t=1,2, \ldots, T$

$\frac{D_{t}^{B}-D_{t}(\bar{X})}{D_{t}^{B}-D_{t}^{L}}+D_{t 2}^{-} \geq 1 ; t=1,2, \ldots, T$

$\bar{X}_{t}-\left(\bar{X}_{t}^{B}-\overline{p_{t}^{-}}\right) \bar{p}_{t}^{-}+\bar{D}_{t 31}^{-} \geq \bar{I} ; \mathrm{t}=1,2, \ldots,(T-1)$

$\left(\bar{X}_{t}^{B}+\bar{p}_{t}^{+}\right)-\bar{X}_{t} \bar{p}_{t}^{+}+\bar{D}_{t 32}^{-} \geq \bar{I} ; t=1,2, \ldots,(T-1)$

$D_{t 1}^{-} \geq 0 ; t=1,2, \ldots, T$

$D_{t 2}^{-} \geq 0 ; t=1,2, \ldots, T$

$\bar{D}_{t 3}^{-} \geq \overline{0} ; t=1,2, \ldots,(T-1)$

$\overline{\mathrm{A}}_{\mathrm{i} 1} \bar{X}_{1}+\overline{\mathrm{A}}_{\mathrm{i} 2} \bar{X}_{2}+$ $+\overline{\mathrm{A}}_{\mathrm{iT}} \bar{X}_{T}(\leq,=, \geq) b_{i}$ $\forall i=1,2, \ldots ., m$

and

$\bar{X}_{1} \geq 0, \bar{X}_{2} \geq 0, \ldots \ldots \ldots, \bar{X}_{T} \geq 0$ 
Here, $D_{t 1}^{-}, D_{t 2}^{-}$are negative deviational variables. $\bar{D}_{t 31}^{-}$and $\bar{D}_{t 32}^{-}$represent the vector of underdeviational variables. $W_{t 1}^{-}=1 /\left(N_{t}^{B}-N_{t}^{L}\right), W_{t 2}^{-}={ }^{-} 1 /{ }_{\left(D_{t}^{B}-D_{t}^{L}\right)}$ and $\bar{W}_{t 31}^{-}=1 /\left(\bar{p}_{t}^{-}\right)$; $\bar{W}_{t 31}^{-}=1 /\left(\bar{p}_{t}^{+}\right) \cdot \bar{I}$ is the column vector having all components equal to 1 , and its dimension depends on $\bar{X}$. By solving Equation 15, if the DMs are satisfied with this solution, then a satisfying solution is reached. Otherwise, higher level DMs should provide new tolerance limits for the control variable until a satisfying solution is reached. In general, considering a set of positive relaxation offered by the higher level DMs, the solution of Equation 15 becomes satisfying for all the level DMs.

\section{Numerical example}

Let us consider the following tri-level linear fractional programming problem as

$\operatorname{Max}_{\mathbf{x}_{1}, x_{2}} Z_{1}=\frac{7 x_{1}+3 x_{2}-4 x_{3}+2 x_{4}}{x_{1}+x_{2}+x_{3}+1}$

$\operatorname{Max}_{x_{3}} Z_{2}=\frac{x_{2}+3 x_{3}+4 x_{4}}{x_{1}+x_{2}+x_{3}+2}$

$\operatorname{Max}_{\mathrm{x}_{4}} Z_{3}=\frac{2 x_{1}+x_{2}+x_{3}+x_{4}}{x_{1}+x_{2}+x_{3}+3}$

subject to

$x_{1}+x_{2}+x_{3}+x_{4} \leq 5$

$x_{1}+x_{2}-x_{3}-x_{4} \leq 2$

$x_{1}+x_{2}+x_{3} \geq 1$

$x_{1}-x_{2}+x_{3}+2 x_{4} \leq 4$

$x_{1}+2 x_{3}+2 x_{4} \leq 3$

$x_{4} \leq 2$

and $x_{1} \geq 0, x_{2} \geq 0, x_{3} \geq 0, x_{4} \geq 0$.

We find the best optimal solution

$N_{1}^{L}=-6$ at $(0,0,1.5,0)$,

$N_{1}^{B}=17$ at $(2.3333,0,0,0.3333)$,

$N_{2}^{B}=9.5$ at $(0,3.5,0,1.5)$,

$N_{2}^{L}=0$ at $(1,0,0,0)$,

$N_{3}^{L}=1$ at $(0,1,0,0)$, and

$N_{3}^{B}=5$ at $(2.3333,0,0.3333,0)$.
Similarly, $D_{1}^{B}=6$ at $(0,3.5,1.5,0)$,

$D_{1}^{L}=2$ at $(1,0,0,0)$,

$D_{2}^{B}=7$ at $(0,3.5,1.5,0)$,

$D_{2}^{L}=3$ at $(1,0,0,0)$,

$D_{3}^{B}=8$ at $(0,3.5,1.5,0)$,

and $D_{3}^{L}=4$ at $(1,0,0,0)$.

Let the first level DM decide that $x_{1}=2.3333$ with -2 (negative) and +2 (positive) tolerance limits and $\mathrm{x}_{2}=0$ with -6.43 (negative) and +6.43 (positive) tolerance limits. Let the second level DM decide that $x_{3}=0$ with -1 (negative) and +1 (positive) tolerance limits.

Then, following the procedure, FGP model I gives the problem as follows:

Minimize $\lambda$ subject to

$\frac{N_{t}(\bar{X})-N_{t}^{L}}{N_{t}^{B}-N_{t}^{L}}+D_{t 1}^{-} \geq 1 ; t=1,2, \ldots, T$
$\Rightarrow 7 x_{1}+3 x_{2}-4 x_{3}+2 x_{4}+23 D_{11}^{-} \geq 17$
$\Rightarrow x_{2}+3 x_{3}+4 x_{4}+9.5 D_{21}^{-} \geq 9.5$
$\Rightarrow 2 x_{1}+x_{2}+x_{3}+x_{4}+4 D_{31}^{-} \geq 5$

$\frac{D_{t}^{B}-D_{t}(\bar{X})}{D_{t}^{B}-D_{t}^{L}}+D_{t 2}^{-} \geq 1 ; t=1,2, \ldots, T$

$\Rightarrow x_{1}+x_{2}+x_{3}-4 D_{12}^{-} \geq 1$

$\Rightarrow x_{1}+x_{2}+x_{3}-4 D_{22}^{-} \leq 1$

$\Rightarrow x_{1}+x_{2}+x_{3}-4 D_{32}^{-} \leq 1$

$\bar{X}_{t}-\left(\bar{X}_{t}^{B}-\overline{p_{t}^{-}}\right) \overline{p_{t}^{-}}+\bar{D}_{t 31}^{-} \geq \bar{I}$

$\left(\bar{X}_{t}^{B}+\bar{p}_{t}^{+}\right)-\bar{X}_{t} \bar{p}_{t}^{+}+\bar{D}_{t 32}^{-} \geq \bar{I} ; t=1,2, \ldots,(T-1)$

$\Rightarrow-0.5 x_{1}+D_{1311}^{-} \geq-1.1666$

$\Rightarrow-0.5 x_{1}+D_{1321}^{-} \geq-1.1666$

$\Rightarrow-0.1555 x_{2}+D_{1312}^{-} \geq 0$

$\Rightarrow-0.1555 x_{2}+D_{1322}^{-} \geq 0$

$\Rightarrow-x_{3}+D_{2311}^{-} \geq 0$

$\Rightarrow-x_{3}+D_{2321}^{-} \geq 0$ 


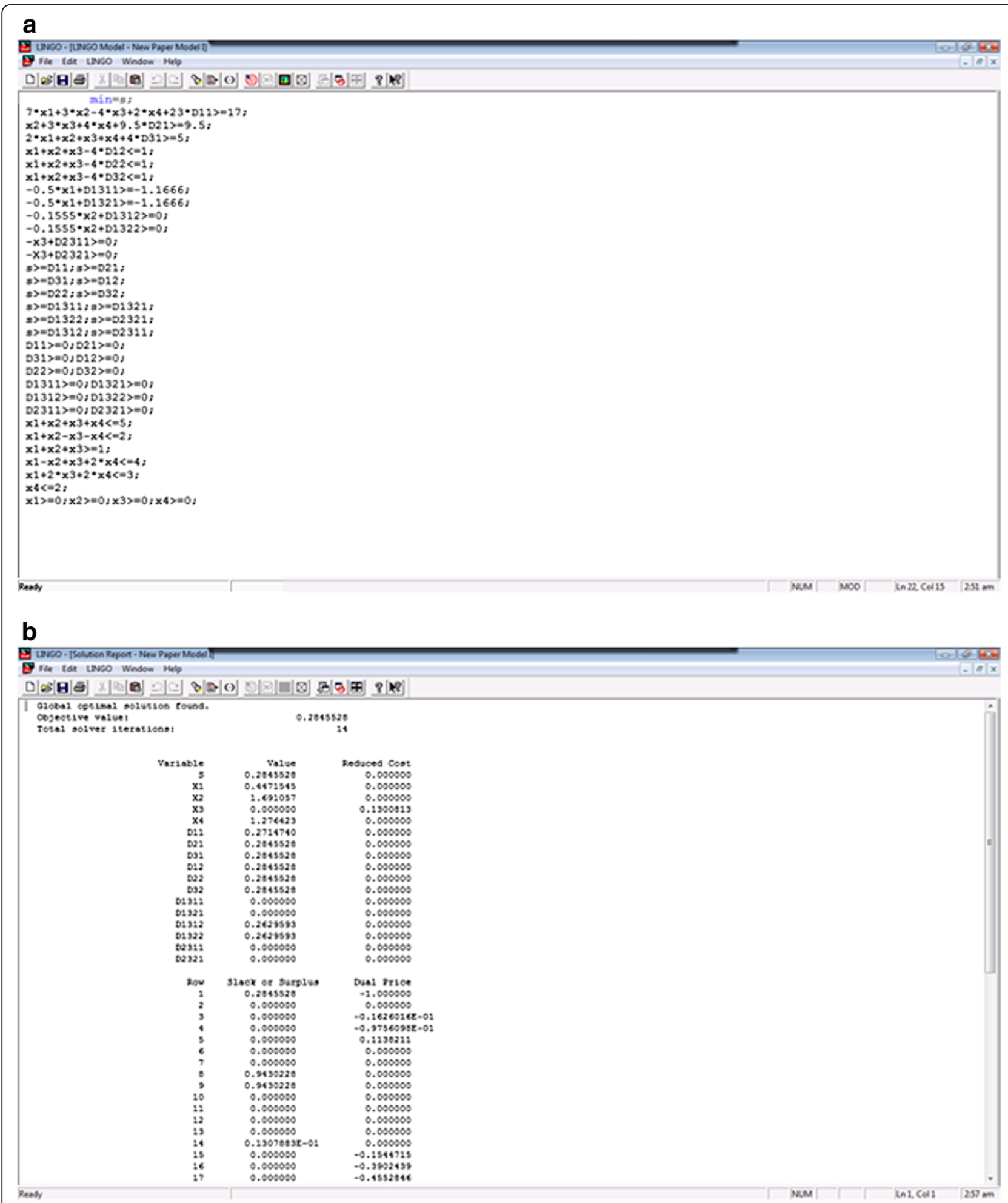

Figure 3 Description (a) and solution (b) of example 1 with model I in LINDO 10.0 (trial version). 
a

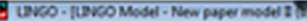

Yile tole unco Whsow melo

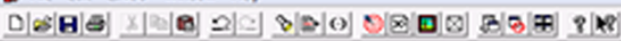

$\mathrm{min}=\mathrm{D} 212+D 22+D 31+D 12+D 22+D 32+D 1312+D 2321+D 1312+D 1322+D 2312+D 2321 ;$

$7 \cdot \times 1+3 \cdot \times 2-4 \cdot \times 3+2 \cdot x 4+23 \cdot 012>-172$

$x 2+3 \cdot x+3+4 \cdot x 4+9.5 \cdot 021>-3.5$

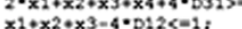

$x 1+x 2+x 3-4 * 022<=1$

$\times 1+\times 2+x 3-4 \cdot D 32<=1$

$-0.5 \cdot \times 1+51311>=-1.1666$

$-0.5 \cdot \times 1+01321>=-1.1666$ :

$-0.1555 \cdot x 2+D 1312>=03$

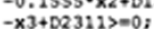

$-\mathrm{x} 3+\mathrm{D} 232 \mathrm{3}>\mathrm{x}=0$

D12> 0 :

D2: $>=0$

D3:> $=0$ :

D12> $=0$

$D 22>0$.

D32> $=02$

D1311>00:

$D 1321>=0$ :

D1312> $=0$,

D1322> $=0$ ?

D2311> $=0$;

$x 1+x_{2}+x^{2}+x^{2}<-5 ;$

$x 1+x 2-x 3-x<<-2 ;$
$x 1+x 2+x 3>-2 ;$

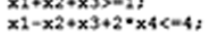

$x 1+2 * x 3+2 * x<<-3 ;$

$x 1>=0 ; \times 2>=0 ; \times 3>=0 ; \times 4>=0$

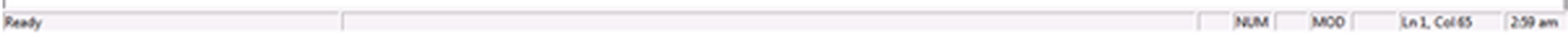

b

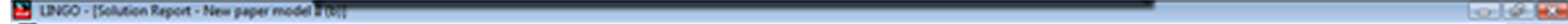

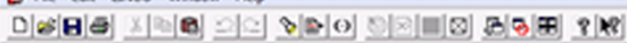

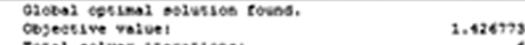

Toral goiver lterotiong,

(2.8.

Variable value nosuced coos

$\begin{array}{lll}011 & 0.3478261 \\ 022 & 0.3703474\end{array}$

$\begin{array}{lll}D 31 & 0.2000000 & 0.000000 \\ 012 & 0.000000 & 0.00000\end{array}$

1.000000

$\begin{array}{lll}032 & 0.2816270 \\ 01312 & 0.000000 & 2.000000\end{array}$

$01922-0.00505001 .000000$

$\begin{array}{lll}0.923 & 0.005050 & 0.0000005 \\ 01922 & 0.005050 & 0.000505\end{array}$

$\begin{array}{lll}52312 & 0.005080 & 0.000000\end{array}$

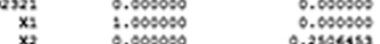

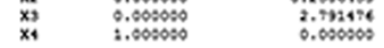

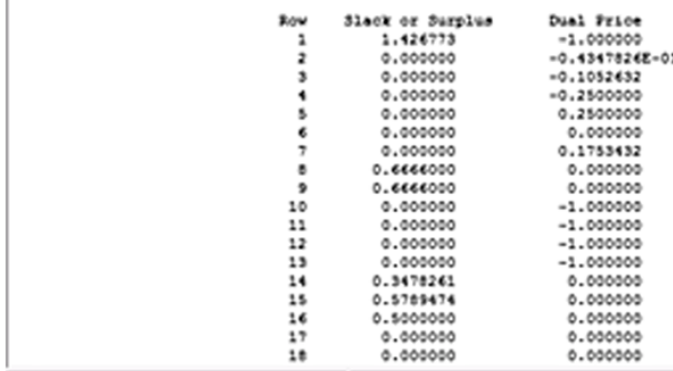

Peasy

0.000000

Figure 4 Description (a) and solution (b) of example 1 with model Ilb in LINDO 10.0 (trial version). 
$\lambda \geq D_{t 1}^{-} ; t=1,2, \ldots, T$

$\Rightarrow \lambda \geq D_{11}^{-}, \quad \lambda \geq D_{21}^{-}, \quad \lambda \geq D_{31}^{-}$

$\lambda \geq D_{t 2}^{-} ; \quad t=1,2, \ldots, T$

$\Rightarrow \lambda \geq D_{12}^{-}, \quad \lambda \geq D_{22}^{-}, \quad \lambda \geq D_{32}^{-}$

$\lambda \geq \bar{D}_{t 31}^{-} \bar{I}, \lambda \geq \bar{D}_{t 32}^{-} \bar{I} ; \quad t=1,2, \ldots,(T-1)$

$\Rightarrow \lambda \geq D_{1311}^{-}, \quad \lambda \geq D_{1321}^{-}, \lambda \geq D_{1312}^{-}$,

$\lambda \geq D_{1322}^{-}, \lambda \geq D_{2311}^{-}, \lambda \geq D_{2321}^{-}$

$D_{t 1}^{-} \geq 0 ; \quad t=1,2, \ldots, T$

$\Rightarrow D_{11}^{-} \geq 0, \mathrm{D}_{21}^{-} \geq 0, \mathrm{D}_{31}^{-} \geq 0$

$D_{t 2}^{-} \geq 0 ; \quad t=1,2, \ldots, T$

$\Rightarrow D_{12}^{-} \geq 0, \mathrm{D}_{22}^{-} \geq 0, \mathrm{D}_{32}^{-} \geq 0$

$\bar{D}_{t 31}^{-} \geq \overline{0}, \bar{D}_{t 32}^{-} \geq \overline{0} ; \quad t=1,2, \ldots,(T-1)$

$\Rightarrow D_{1311}^{-} \geq 0, \mathrm{D}_{1321}^{-} \geq 0, \mathrm{D}_{1312}^{-} \geq 0, \mathrm{D}_{1322}^{-} \geq 0$,

$\mathrm{D}_{2311}^{-} \geq 0, \mathrm{D}_{2321}^{-} \geq 0$

$\overline{\mathrm{A}_{\mathrm{i} 1}} \quad \overline{X_{1}}+\overline{\mathrm{A}_{\mathrm{i} 2}} \quad \overline{X_{2}}+$ $+\overline{\mathrm{A}_{\mathrm{iT}}} \quad \overline{X_{T}}(\leq,=, \geq) b_{i}$

$\Rightarrow x_{1}+x_{2}+x_{3}+x_{4} \leq 5$ $\forall i=1,2, \ldots ., m$

$x_{1}+x_{2}-x_{3}-x_{4} \leq 2$

$x_{1}+x_{2}+x_{3} \geq 1$

$x_{1}-x_{2}+x_{3}+2 x_{4} \leq 4$

$x_{1}+2 x_{3}+2 x_{4} \leq 3$

$x_{4} \leq 2$

and $\bar{X}_{1} \geq 0, \bar{X}_{2} \geq 0, \ldots \ldots \ldots, \bar{X}_{T} \geq 0$

$\Rightarrow x_{1} \geq 0, x_{2} \geq 0, x_{3} \geq 0, x_{4} \geq 0$.

Solving the above programming problem using nonlinear packages (as shown in Figure 3a,b), we obtain solution $x_{1}=0.4471, \quad x_{2}=1.69105, x_{3}=0.0000$, and $x_{4}=1.2764, \quad \lambda=0.2845 ;$ and $Z_{1}=3.42738, \quad Z_{2}=$ 1.642437, and $Z_{3}=0.7515643$.

Model IIb: Find $\bar{X}$ so as to minimize

$\lambda=\sum_{t=1}^{T} D_{t 1}^{-}+\sum_{t=1}^{T-1} D_{t 2}^{-}+\sum_{t=1}^{T-1} \bar{D}_{t 3}^{-}$

$\min \lambda=\left(D_{11}^{-}+D_{21}^{-}+D_{31}^{-}\right)+\left(D_{12}^{-}+D_{22}^{-}+D_{32}^{-}\right)$ $+\left(D_{1311}^{-}+D_{2311}^{-}+D_{1321}^{-}+D_{1312}^{-}+D_{2321}^{-}+D_{1322}^{-}\right)$ subject to

$$
\begin{aligned}
& \mu_{Z_{t}}\left(N_{t}(\bar{X})\right)+D_{t 1}^{-} \geq 1 ; t=1,2, \ldots, T \\
& \Rightarrow 7 x_{1}+3 x_{2}-4 x_{3}+2 x_{4}+23 D_{11}^{-} \geq 17 \\
& \Rightarrow x_{2}+3 x_{3}+4 x_{4}+9.5 D_{21}^{-} \geq 9.5 \\
& \Rightarrow 2 x_{1}+x_{2}+x_{3}+x_{4}+4 D_{31}^{-} \geq 5 \\
& \mu_{Z_{t}}\left(D_{t}(\bar{X})\right)+D_{t 2}^{-} \geq 1 ; t=1,2, \ldots, T \\
& \Rightarrow x_{1}+x_{2}+x_{3}-4 D_{12}^{-} \geq 1 \\
& \Rightarrow x_{1}+x_{2}+x_{3}-4 D_{22}^{-} \leq 1 \\
& \Rightarrow x_{1}+x_{2}+x_{3}-4 D_{32}^{-} \leq 1 \\
& \mu_{\bar{X}_{t}}\left(\bar{X}_{t}\right)+\bar{D}_{t 3}^{-} \geq \bar{I} ; \quad t=1,2, \ldots,(T-1) \\
& \bar{X}_{t}-\left(\bar{X}_{t}^{B}-\overline{p_{t}^{-}}\right) \overline{\overline{p_{t}^{-}}}+\bar{D}_{t 31}^{-} \geq \bar{I} ; \\
& \left(\bar{X}_{t}^{B}+\bar{p}_{t}^{+}\right)-\bar{X}_{t} \bar{p}_{t}^{+}+\bar{D}_{t 32}^{-} \geq \bar{I} ; t=1,2, \ldots,(T-1) \\
& \Rightarrow-0.5 x_{1}+D_{1311}^{-} \geq-1.1666 \\
& \Rightarrow-0.5 x_{1}+D_{1321}^{-} \geq-1.1666 \\
& \Rightarrow-0.1555 x_{2}+D_{1312}^{-} \geq 0 \\
& \Rightarrow-0.1555 x_{2}+D_{1322}^{-} \geq 0 \\
& \Rightarrow-x_{3}+D_{2311}^{-} \geq 0 \\
& \Rightarrow-x_{3}+D_{2321}^{-} \geq 0 \\
& D_{t 1}^{-} \geq 0 ; \quad t=1,2, \ldots, T \\
& \Rightarrow D_{11}^{-} \geq 0, \mathrm{D}_{21}^{-} \geq 0, \mathrm{D}_{31}^{-} \geq 0 \\
& D_{t 2}^{-} \geq 0 ; \quad t=1,2, \ldots, T \\
& \Rightarrow D_{12}^{-} \geq 0, \mathrm{D}_{22}^{-} \geq 0, \mathrm{D}_{32}^{-} \geq 0, \bar{D}_{t 3}^{-} \geq \overline{0} ; \quad t=1,2, \ldots,(T-1) \\
& \Rightarrow D_{1311}^{-} \geq 0, \mathrm{D}_{1321}^{-} \geq 0, \mathrm{D}_{1312}^{-} \geq 0 \text {, } \\
& \mathrm{D}_{1322}^{-} \geq 0, \mathrm{D}_{2311}^{-} \geq 0, \mathrm{D}_{2321}^{-} \geq 0 \\
& \forall i=1,2, \ldots . m
\end{aligned}
$$

Solving the above FGP model problem using nonlinear packages (as shown in Figure 4a,b), we obtain solution $x_{1}=1.0000, x_{2}=0.0000, x_{3}=0.0000$, and $x_{4}=1.0000$, 
Table 1 Comparison of euclidean distance for the solution of example 1

\begin{tabular}{lllll}
\hline Method & $\left(\boldsymbol{x}_{1}, \boldsymbol{x}_{2}, \boldsymbol{x}_{3}, \boldsymbol{x}_{4}\right)$ & $\left(\boldsymbol{Z}_{1}, \boldsymbol{Z}_{2}, \boldsymbol{Z}_{3}\right)$ & $\left(\mu_{\mathbf{z}_{1}}\left(\boldsymbol{N}_{1}(\overline{\boldsymbol{X}}), \mu_{\mathbf{z}_{1}}\left(\boldsymbol{D}_{1}(\overline{\boldsymbol{X}}), \mu_{\mathbf{z}_{2}}\left(\boldsymbol{N}_{\mathbf{2}}(\overline{\boldsymbol{X}}), \mu_{\mathbf{z}_{2}}\left(\boldsymbol{D}_{2}(\overline{\boldsymbol{X}}), \mu_{\mathbf{z}_{3}}\left(\boldsymbol{N}_{3}(\overline{\boldsymbol{X}}), \mu_{\mathbf{z}_{3}}\left(\boldsymbol{D}_{3}(\overline{\boldsymbol{X}})\right)\right.\right.\right.\right.\right.\right.$ & Euclidean distance \\
\hline Proposed & $(0.4471,1.69105$, & $(3.42738,1.642437$, & $(0.7285,0.7155,0.7154,0.7155,0.7154,0.7154)$ & 0.6918 \\
method I & $0.0000,1.2764)$ & $0.7515643)$ & & 0.706163 \\
Proposed & $(1.0000,0.0000$, & $(4.5,1.3333,0.75)$ & $(0.6521,1,0.42105,1,0.5000,1)$ & \\
method IIb & $0.0000,1.0000)$ & & &
\end{tabular}

Based on proposed FGP methods I and Ilb.

Table 2 Solution set (for example 1) based on the tolerance on the decision variables for model

\begin{tabular}{lllll}
\hline$\left(\boldsymbol{p}_{1}^{-}, \boldsymbol{p}_{1}^{+}\right)$ & $\left(\boldsymbol{p}_{1}^{-}, \boldsymbol{p}_{1}^{+}\right)$ & $\left(\boldsymbol{p}_{2}^{-}, \boldsymbol{p}_{2}^{+}\right)$ & $\left(\boldsymbol{x}_{1}, \boldsymbol{x}_{2}, \boldsymbol{x}_{3}, \boldsymbol{x}_{4}, \lambda\right)$ & $\left(\boldsymbol{Z}_{1}, \boldsymbol{Z}_{2}, \boldsymbol{Z}_{3}\right)$ \\
\hline$(-2,2)$ & $(-6.43,6.43)$ & $(-1,1)$ & $(0.4471,1.69105,0.0000,1.2764,0.2845)$ & $(3.42738,1.642437,0.7515643)$ \\
$(-2,2)$ & $(-5.5,5.5)$ & $(-1,1)$ & $(0.46383,1.623581,0.0000,1.268083,0.295167)$ & $(3.450697,1.638180,0.7507402)$ \\
$(-2,2)$ & $(-5,5)$ & $(-1,1)$ & $(0.48427,1.54088,0.0000,1.25786,0.3081761)$ & $(3.480241,1.632814,0.7496851)$ \\
$(-2,2)$ & $(-4.5,4.5)$ & $(-1,1)$ & $(0.50655,1.450743,0.0000,1.24672,0.322355)$ & $(3.513862,1.626774,0.7485059)$ \\
$(-2,2)$ & $(-4,4)$ & $(-1,1)$ & $(0.5310345,1.351724,0.0000,1.234481,0.337931)$ & $(3.552630,1.619892,0.7471748)$ \\
\hline
\end{tabular}

$\lambda=1.426773 ;$ and $Z_{1}=3.42738, Z_{2}=1.642437$, and $Z_{3}=0.7515643$ (see Table 1 for a comparison of Euclidean distance and Table 2 for the solution set of example 1).

\section{Conclusion}

An effort has been made to solve the multilevel fractional programming problem based on the fuzzy set theory and goal programming approach. The main advantage of the proposed approach is that computational complexity is reduced by defining separate membership functions for numerator and denominator functions of objectives at each level, but tolerance values (positive and negative) are chosen so that the satisfying solution is forced towards the optimal solutions. However, the main difficulties of these methods are the following: (1) Preference information required from the DM cannot easily be given, particularly when the information thus required is large and complex and/or the objective functions in fractional form are measured on different scales, and (2) if DMs are not satisfied with solution, then higher level DMs should provide new tolerance limits for the control variables until a satisfying solution is reached.

Besides this, the proposed methodology can be easily reduced to the solution procedure as given by Sinha (2003b) and Pramanik and Roy (2007) for the multilevel linear programming case by avoiding the goal membership functions for denominators of objectives and corresponding goal variables.

\section{Methods}

\section{Selection of compromise solution}

The concept of utopia point (the ideal point) and the use of distance function for group decision analysis was first studied by Yu (1973). Biswas and Pal (2005) used the Euclidean distance function to select the appropriate priority structure in the application of fuzzy goal programming technique to land use planning in the agricultural system. Here, different FGP models provide different optimal solutions. The Euclidean distance function is used only to identify which FGP model (model I, model IIa, and model IIb) offers a better optimal solution. In the FGP formulation, since the aspired level of each of the membership function goals is unity, the point consisting of the highest membership value of each of the goals would represent the ideal point. The Euclidean distance function can be defined in this case as follows:

$D^{2}=\left[\sum_{t=1}^{T}\left\{\left[1-\mu_{Z_{t}}\left(N_{t}\left(\bar{X}_{t}\right)\right]^{2}+\left[1-\mu_{Z_{t}}\left(D_{t}\left(\bar{X}_{t}\right)\right]^{2}\right\}\right]^{1 / 2}\right.\right.$

where $\mu_{Z_{t}}\left(N_{t}\left(\bar{X}_{t}\right)\right.$ and $\mu_{Z_{t}}\left(D_{t}\left(\bar{X}_{t}\right)\right.$ represent the achieved membership value of the $\mathrm{t}$-th numerator and denominator objective goals, respectively. The solution for which $D^{2}$ is minimum would be the most satisfying solution.

\section{Competing interests}

The authors declare that they have no competing interests.

\section{Authors' contributions}

$\mathrm{KL}$ carried out discussion related to formulation of MLFPP, related terminology, Characterization of linear membership functions for the numerator and denominator functions at each level as well as decision vector. KL proposed FGP approach to tackle MLFPPs and formulate different mathematical models related to it and also illustrate proposed methodology with a numerical example and show sensitivity analysis performed on it. MPP described the use of selection criteria of compromise solution for MLFPPS. All authors read and approved the final manuscript.

\section{Acknowledgements}

The authors are indebted to the anonymous referee for the encouraging comments and valuable suggestions, which have improved the presentation of the paper. 


\section{Author details}

${ }^{1}$ Department of Mathematics, Government Engineering College, Bikaner 334004, India. ²Department of Mechanical Engineering, Government Engineering College, Bikaner 334004, India.

Received: 10 March 2010 Accepted: 2 March 2012

Published: 23 August 2012

\section{References}

Anandilingam G (1988) A mathematical programming model of decentralized multilevel system. J Oper Res Soc 39(11):1021-1033

Anandilingam G, Apprey V (1991) Multi-level programming and conflicting resolution. European Journal of Operational Research 51:233-247

Arora SR, Gupta R (2009) Interactive fuzzy goal programmings approach for bilevel programming problem. European Journal of Operational Research 194:368-376

Baky IA (2009) Fuzzy goal programming algorithm for solving decentralized bilevel multiobjective programming problems. Fuzzy Sets and Systems 160:2701-2710

Bellmann RE (1957) Dynamic programming. Princeton University Press, Princeton

Biswas A, Pal BB (2005) Application of fuzzy goal programming technique to land use planning in agriculture systems. Omega 33:391-398

Chakraborty M, Gupta S (2002) Fuzzy mathematical programming for multi objective linear fractional programming problem. Fuzzy Sets and Systems 125:335-342

Chang CT (2009) A goal programming approach for fuzzy multiobjective fractional programming problems. International Journal of System Sciences 40:867-874

Charnes A, Cooper WW (1962) Programming with linear fractional functional. Nov Res Logistics Quart 9:181-186

Craven BD, Mond B (1975) On fractional programming and equivalence. Nov Res Logistics Quart 22:405-410

Jimenez M, Bilbas A (2009) Pareto optimal solutions in fuzzy multi-objective linear programming. Fuzzy Sets and Systems 160:2714-2721

Lai YJ (1996) Hierarchical optimization: a satisfactory solution. Fuzzy Sets and Systems 77:321-335

Lai YJ, Hwang CL (1993) Fuzzy mathematical programming methods and applications. Springer, Berlin

Mohamed RH (1997) The relationship between goal programming and fuzzy programming. Fuzzy Sets and Systems 89:215-222

Pal BB, Gupta S (2009) A genetic algorithm to fuzzy goal programming formulation of fractional multiobjective decision making problem. In: Proceeding of 1st International Conference on Advanced Computing (ICAC). Chennai, 13-15 December 2009

Pramanik S, Roy TK (2007) Fuzzy goal programming approach to multilevel programming problems. European Journal of Operational Research 176:1151-1166

Shih HS, Lee ES (2000) Compensatory fuzzy multiple decision making. Fuzzy Sets and Systems 14:71-87

Shih HS, Lai YJ, Lee ES (1996) Fuzzy approach for multi-level programming problems. Computer and Operation Research 23:73-91

Sinha S (2003a) Fuzzy mathematical programming applied to multi-level programming problems. Computers \& Operations Research 30:1259-1268

Sinha S (2003b) Fuzzy programming approach to multi-level programming problems. Fuzzy Sets and Systems 136:189-202

Yu PL (1973) A class of solutions for group decision problems. Management Sciences 19:936-946

doi:10.1186/2251-712X-8-16

Cite this article as: Lachhwani and Poonia: Mathematical solution of multilevel fractional programming problem with fuzzy goal

programming approach. Journal of Industrial Engineering International 2012, 8:16

\section{Submit your manuscript to a SpringerOpen ${ }^{\circ}$ journal and benefit from:}

- Convenient online submission

- Rigorous peer review

- Immediate publication on acceptance

- Open access: articles freely available online

- High visibility within the field

- Retaining the copyright to your article

Submit your next manuscript at $>$ springeropen.com 